\title{
New double indentation technique for measurement of the elasticity modulus of thin objects
}

\author{
Joris A.M. Soons • Ives de Baere • Joris J.J. Dirckx
}

Received: Oct 02, 2009 / Accepted: Feb 16, 2010

\begin{abstract}
In this paper we introduce a new method to determine the Young's modulus of thin (biological) samples. The method is especially suitable for small objects with a thickness of a few hundred micrometers. Such specimens cannot be examined with existing tests: compression and tensile tests need well-known geometry and boundary conditions while classic indentation tests need relatively thick pieces of material. In order to determine the elastic modulus we use the indentation theory as proposed by Sneddon and correct it with a finite element calculated $\kappa$ factor to compensate for the small thickness. In order to avoid material deformations at the contact zone between the sample bottom and the sample stage, we replace the sample stage by a second indentation needle. In this way the sample can be clamped between two identical needles and a virtual mirror plane is introduced. The new method was used on four test-materials and results agreed well with the outcome of a standard compression method applied on large samples of the same materials. As an application example the technique was applied on thin biological samples, namely middle ear ossicles of rabbits.
\end{abstract}

Keywords double indentation · elasticity modulus · thin objects - FE modelling - measurement method · middle ear ossicle bone

J. Soons and J. Dirckx

Laboratory of Biomedical Physics, University of Antwerp, Groenenborgerlaan 171, B-2020 Antwerp, Belgium

Tel.: +32-3-2653438

Fax: +32-3-2653318

E-mail: joris.soons@ua.ac.be

I. de Baere

Materials Science and Engineering, Ghent University, SintPietersnieuwstraat 41, B-9000 Ghent, Belgium

\section{Introduction}

Finite element (FE) models are widely used to investigate (bio-)mechanical problems. However, to create accurate models, the exact geometry, the boundary conditions and the material properties of the components have to be known precisely [1]. A very important parameter for (linear elastic) materials is the Young's modulus $(E)$. For large material samples, standard tensile and compression methods for measuring Young's moduli are widely available (e.g. ASTM D695-02a 'Standard Test Method for Compressive Properties of Rigid Plastics'). Those methods are very useful to determine the modulus of larger biomaterials, like the femur bone [2]. These tests require a precise description of the geometry of the specimen and usually a cylinder or a cuboid needs to be created. In order to avoid slipping or stretching, the boundary conditions at the contact zone have to be controlled very well. However, when applying this technique on small materials, these conditions are that difficult to realize that even a very fine preparation cannot avoid inaccuracies [3, 4].

Another way to obtain material parameters is through backwards engineering: the material properties in the model are changed to get a best fit between simulation and experiment. Although good results on small specimens can be obtained, this technique needs unique experiments and time-consuming simulations [5, 6, 7]. As an alternative, indentation testing can provide the material properties. Originally, these tests only give the hardness, an empirical number which represent the difficulty of cutting in the material. The hardness is not a real physical entity, so it is unusable for simulations. A method to obtain the elastic modulus, from those hardness indentator experiments, is based on classical Hertz contact mechanics and was proposed by Sneddon 
[8]. This technique is applied widely in material and biomechanical sciences, at microscale $[4,9,10,11,12$, $13,14,15]$ and nanoscale $[16,17,18,19]$. Such tests use different punches (cylindrical, conical, Berkovich, Vickers) and different loading protocols [11, 15, 20]. Oliver and Pharr [20] found that the unloading-displacement relationships from the indentation experiment could be described by:

$P=\alpha h^{m}$

In this equation $P$ is the indenter load, $h$ is the elastic displacement of the indenter and $\alpha$ and $m$ are constants. The value of $m$ depends on the punch geometries ( $m=1$ for flat cylinders). According to Sneddon [8], the elastic modulus for a rigid body punch can be obtained by:

$E=\frac{\sqrt{\pi}}{2} \cdot \frac{S \cdot\left(1-\nu^{2}\right)}{\sqrt{A}}$

In this equation, $E$ represents the elastic modulus, $A$ is the contact area, $\nu$ is the Poisson's ratio of the material and $S$ is the measured stiffness. The stiffness $S$, given by $d P / d h$, is obtained from the unloading curve which is, as seen in equation 1, linear for cylindrical punches $(m=1 \Rightarrow S=\alpha)$.

The method as described above was theoretically derived for a rigid punch which infinitesimally indents a half space elastic material. As a result, it is only valid for relatively thick materials (in comparison with the radius of the indenter), and not for thin samples. Decreasing the indentersize to measure such thinner specimens is not always a good solution, because it is important to use dimensions which represent the realistic loading (micro/nano versus macro effects), especially for biomaterials. A nano-indentation on bone for instance, gives information about the micromechanics (fibers and cells) and not about the macroscopic bulk properties [21]. For that reason, Hayes et al. [22] extended the solution of Sneddon [8] to measure the Young's modulus of a thin cartilage layer fixed on bone. They calculated a theoretical correction factor $\kappa$ which compensates the mismatch for such a thin material fixed on a rigid body. The corrected formula for a cylindrical punch becomes:

$E=\frac{\sqrt{\pi}}{2} \cdot \frac{S \cdot\left(1-\nu^{2}\right)}{\sqrt{A}} \cdot \frac{1}{\kappa}$

To apply this technique on freestanding thin materials, we introduce two modifications to the existing theory. First we will calculate a new $\kappa$ for thin materials laying on a rigid body without friction, instead of being fixed to it. For this purpose we create a FE model of the indentation experiment. Secondly, when a force is applied, thin materials will be easily deformed on large plane contacts, so the contact-dependent deformation is measured instead of the true indentation deformation. In order to avoid deformation on an extended and poorly controlled contact surface, a second indentation needle is introduced.

In this paper we will validate our new method on four test-materials with different thickness by comparing the results from the new method to a standard compression test (ASTM D695-02a). Finally, we will apply our method on biological samples and we will determine the elasticity modulus of middle ear (ME) ossicle bone. In current modelling of middle ear mechanics, those bones are treated either as rigid bodies or standard values for the Young's modulus are used (12 $\pm 3 \mathrm{GPa})[23,24]$. A correct value of the elasticity modulus becomes very important when bending of the auditory ossicles needs to be taken into account. Quasi-static pressure variations, such as atmospheric pressure variations, can be several orders of magnitude larger than the loudest sound pressures and ossicle bending may become important in this case. In the high frequency range ossicle bending occurs due to inertia effects and it will influence the middle ear transfer function $[25,26]$.

\section{Experimental procedure}

\subsection{Experimental setup}

Figure 1 illustrates the setup. Two identical flattened cones were placed exactly opposite to one another so that a thin material could be clamped between them (1). The cones were custom-made from high speed steel (HSS) and had a top angle of $25^{\circ}$ (angle to central axis). The point of the cones were polished to obtain a circular and flat contact zone. The exact radius of these surfaces was measured with a calibrated microscope (Zeiss LSM510 Meta laser scanning microscope) and was $60.8 \pm 1.2 \mu \mathrm{m}$. The Young's modulus of HSS is $210 \mathrm{GPa}$ which is significantly higher than the materials to be tested. The upper cone was attached to a piezo-transducer (PI P-841.60) (2) and a vertical translation stage (3). The piezo-transducer had an embedded strain gage with an active feedback loop, so the displacements were controlled to an accuracy better than $50 \mathrm{~nm}$. Larger vertical translations, needed to bring the needle in position, were executed by a mechanical translation stage. The position of this translation stage could be read from the digital micrometer (4) and was used to measure the thickness of the sample. The bottom needle was attached to a loadcell (5) (Sensotec model $31,50 \mathrm{~N}$ range), which could be translated (6) in the 


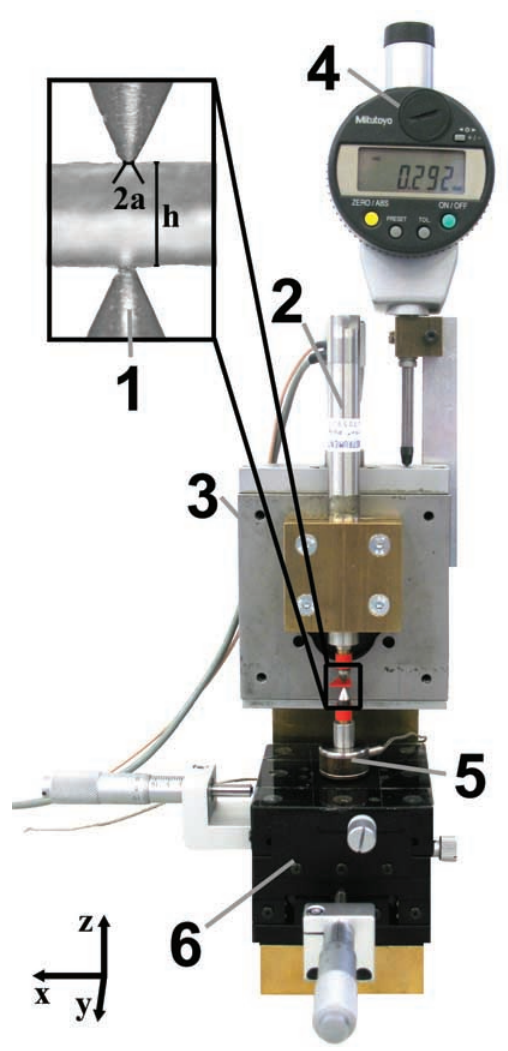

Fig. 1 Picture of the experimental setup showing the flattened cone(1), piezo-transducer to apply displacement (2), vertical translation stage (3), micrometer(4), loadcell(5) in plane translation stage(6)

horizontal plane in order to bring the two needles precisely into line, thus forming a virtual horizontal mirror plane in between the two needles. The whole process was observed with a stereo light microscope. In order to control the piezo displacements and to obtain displacement- and force-data, a Matlab program, interfacing with an A/D-D/A board in a personal computer (National Instruments Daqpad-6020E, 12bit multifunction I/O), was developed.

\subsection{Calibration and loading protocol}

Should the indentation test have been performed with a perfect experimental setup and on a perfectly linear, isotropic and homogenous material, a typical relation between indentation depth and reaction force will be found. This curve, with slightly increasing stiffness for increasing indentation, can be found from FEsimulation, dealing with geometric nonlinearity, and is the same for loading and unloading $[4,10]$. For real measurements, however, there will be some factors, such as nonlinear material behavior and inaccuracies of the experimental setup which will influence this ideal result.
We minimized these factors by doing a calibration and using a proper loading protocol as follows.

First, the loadcell has a rather high compliance as compared to the materials under test, so the measured displacement distances were significantly larger than the actual indentation. The deformation of the loadcell is however solely dependent on the force which is applied to it, which means we could compensate this effect with a calibration. In order to perform the calibration the needles were pushed against each other without a sample in between. Seeing there was no testing material between the needles the force measured on the loadcell corresponded to the deformation of the loadcell. Actually, not only the loadcell's compression was corrected, also all smaller deformations of the entire setup were taken into account.

Second, the indentation points themselves were no perfect rigid bodies, as assumed in equation 2, consequently, they also deformed slightly. This deformation is mainly determined by the indentation force, but also by the compliance of the material used under test. Which brought us to choosing a very stiff material and a cone shape for the indentation points, this meant that their deformation was much smaller than the deformation of the material under test and the bending of the needle itself was minimized.

Next, the needle and the material surface were not perfectly flat, so the contact-surface may not be constant as assumed in equation 1 . We performed a FE simulation of this situation using the FE software package FEBio [27], without dealing with nonlinear phenomena. We found that the stiffness will not rise when the needle penetrated 1.2 times the variation on the contactsurface height. Figure 2 shows plots of the indentation force and the calculated material stiffness when we simulated an indentation using a slightly inclined flat punch, with offset angles between 0 and $3.5^{\circ}$. The values are given as a function of the ratio of the nonflatness measure of the indentation interface and the indentation depth. As we can see from these graphs, a steady value was obtained once this ratio equals 1.2. 


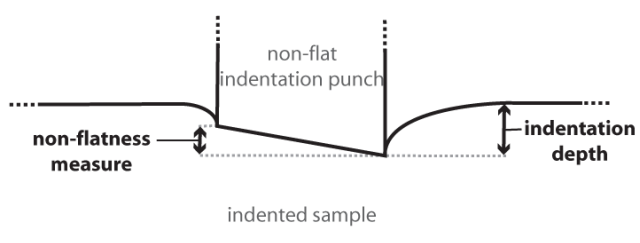

(a)

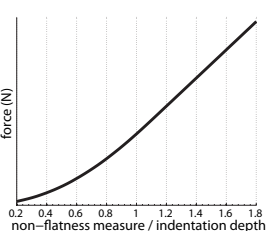

(b)

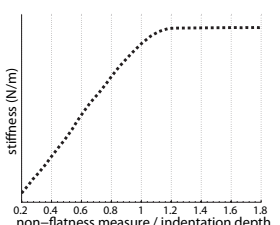

(c)
Fig. 2 (a) Exaggerated cross section of a situation where the interface between the indentation punch and the sample surface is not flat. The definition of non-flatness measure and indentation depth are indicated. A FE model of this situation was used to investigate the effect of the non-flatness.(b) indentation force found in this model as a function of the ratio of the non-flatness measure and the indentation depth. (c) Material stiffness obtained from the model as a function of this ratio.

Finally, real materials do not have a perfect linear elastic behavior and visco-elasticity induces creep and relaxation, so pre-conditioning is necessary. A testing protocol was developed and is presented in figure 3. A triangular wave function drove the piezo to apply 50 preloading indentation cycles. The piezo-displacement and the loadcell reaction force were recorded. After using a median filter with a window of $0.012 s$, a linear fit was made to the piezo-displacement and the reaction force curve (to the middle part of this curve). Application of the calibration gave the effective indentation and so the stiffness could be calculated. The amplitude and period of the piezo-displacement were chosen in such a way that the effective indentation was approximately $1 \mu \mathrm{m}$ and the effective unloading speed was $1 \mathrm{~mm} / \mathrm{min}$. Due to relaxation effects, material inertia and filtering, the resulting displacement and force curves were smoothed. We used this protocol repeatedly, with increasing indentation depth (offsets between 5 and $10 \mu \mathrm{m}$ ), but with approximately the same loading/unloading speed and indentation amplitude. As such, we obtained the stiffness and the modulus (equation 2) with increasing indentation depth and we minimized the effect of stiffening and the effect of non-flatness of the needle contact.

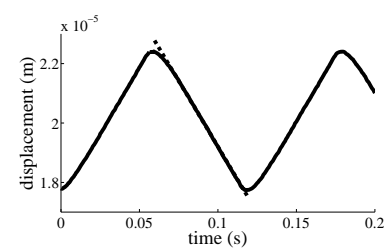

(a) piezo-displacement

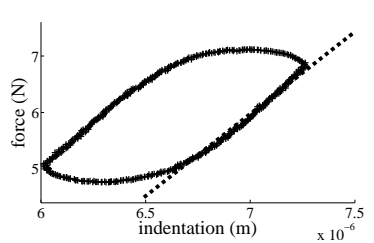

(c) effective indentation vs force

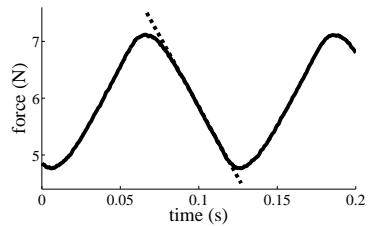

(b) reaction force

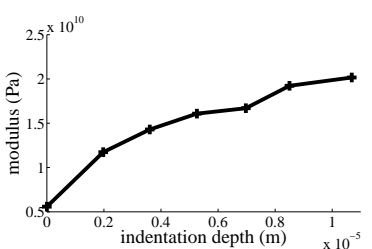

(d) modulus
Fig. 3 (a) and (b): piezo-displacement and loadcell reaction force as a function of time. The dotted line represents the linear fit used in the calculations. (c): the indentation force as a function of the effective indentation depth (both smoothed with median filter). The dotted line represent the stiffness $(d F / d \omega)$ calculated with the linear fit obtained from (a) and (b). (d): calculated modul for different indentation depths.

\section{$2.3 \mathrm{FE}$ calculations for $\kappa$}

Equation 2 as proposed by Sneddon [8], gave the Young's modulus for an infinitesimally indented half-space linear elastic material, which is homogenous and isotropic. In order to investigate thin cartilage layers, fixed on bone, Hayes et al. [22] introduced a correction factor $\kappa$ which compensates for thin materials:

$E=\frac{E_{\text {Sneddon }}}{\kappa(a / h, \nu)}$

where $E$ is the corrected modulus, $E_{\text {Sneddon }}$ is the modulus calculated with equation 2 and $\kappa$ is the correction factor, which depends on the aspect ratio of the indenter radius $(a)$ and the material thickness $(h)$ and on the Poisson's ratio. The face opposite to the indented surface was fixed to a rigid body (bone). We want to obtain the Young's modulus for a material with a perfect sliding contact between the thin sample and a rigid body (sample stage), so a new $\kappa$ had to be calculated. We obtained this factor by finite element calculations performed in FEBio [27]. As seen on figure 4, we used the symmetry of the problem to reduce the amount of elements with a factor of 8 . The maximum number of elements was approximately 50000 and a linear, tetrahedral element was used. The test was also done with less elements to test the sensitivity of the $\kappa$-value calculation to mesh density. 


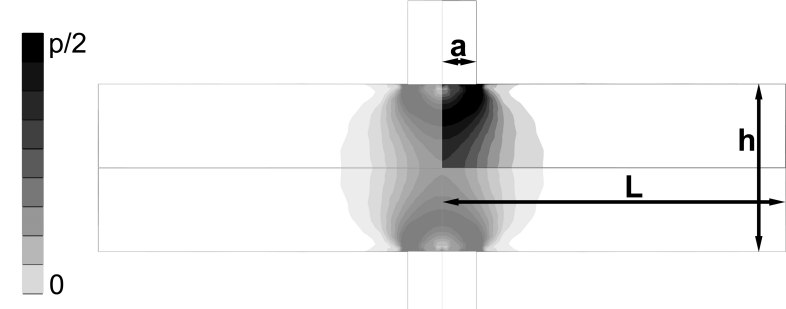

Fig. 4 Cross section of the FE results: for the transparent parts the solution is not calculated but obtained from symmetry, $a$ is the radius of the indenter, $h$ the thickness of the material and $L$ is the dimension/radius of the material surface. Darker colors represent higher Von Mises stresses

The model had an adaptable indenter radius, material dimensions, Poisson's ratio and Young's modulus. We used a Young's modulus $E$ in the model and calculated $E_{\text {Sneddon }}$ (in the model) using equation 2 . The ratio of $E_{\text {Sneddon }}$ and $E$ gave the value for $\kappa$ (see equation 4). Repeating this simulation for different inputvalues learnt us that $\kappa$ depends on the Poisson's ratio $(\nu)$ and on the aspect ratio of the indenter radius and the material thickness $(a / h)$, which is the same as in the model from Hayes et al. [22].

Simulating and interpolating for a whole range of Poisson's ratios and aspect ratios (indenter radius versus material thickness) produced the curves shown in figure 5. The difference between the two graphs were the boundary conditions for the needle. In the left figure the contact zone between the needle and material surface was fixed, while the right figure used a frictionless sliding contact.

\subsection{Validation materials and compression test}

In order to validate our new test setup, we compared the material parameters obtained from experiments with our new indentation setup with those of a standardised test method. We performed indentation tests on thin samples of different materials of which large samples were also available. Four materials were selected, namely Aluminium ( $\mathrm{Al}$ ), Polyvinyl Chloride (PVC), Polymethyl methacrylate or acrylic glass (PMMA) and polycaprolactam or nylon 6 (PA6). These materials samples span a large range of Young's moduli $( \pm 2 G P a$ to $\pm 60 \mathrm{GPa}$ ). All experiments on the large samples were performed according to the ASTM D695-02a 'Standard Test Method for Compressive Properties of Rigid Plastics'. According to this standard, the specimens were milled to cyclinders with a diameter of $12.7 \mathrm{~mm}$ and a height of $25.4 \mathrm{~mm}$.

All experiments were done on an elektromechanical Instron tensile testing machine with a FastTrack 8800 dig- ital controller and a $10 k N$ or $100 k N$ loadcell, depending on the material. All tests were done in a displacementcontrolled mode using a displacement speed of $1 \mathrm{~mm} / \mathrm{min}$, corresponding to the test speed in our indentation method. As such, time-dependent material effects did not differ between the two types of experiments. The cylindrically shaped specimen was placed in the centre of the two cylindrical pressure plates (see figure 6). In order to accurately measure the displacement of the discs, a linear variable differential transformer (LVDT) was used. As such, the deformation of the tensile machine was not taken into account.

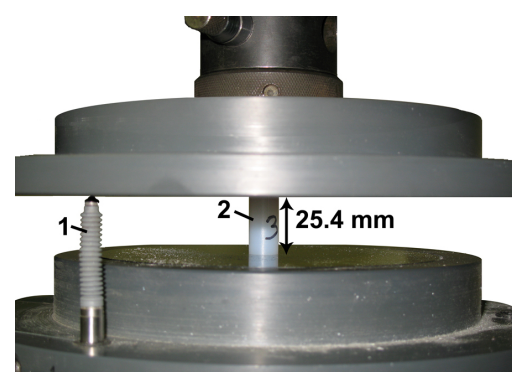

Fig. 6 Compression method: linear variable differential transformer (LVDT) (1) and test material (2).

Since friction between the specimen and the pressure plate may have an important influence on the results, teflon spray was used between specimen and plate, so that the friction may be neglected for the lower load levels, used to calculate Young's modulus. Several experiments were conducted on different specimens of the same material and the stiffness and standard deviation were calculated according to the ASTM norm. The results of these experiments are given in section 3.1.

\subsection{Biological samples: middle ear ossicles}

As a demonstration, we applied our method to measure the Young's modulus of auditory ossicles. Middle ear ossicles were harvested from young-adult, male New Zealand white rabbits immediately after sacrificing the animal by injection of $120 \mathrm{mg} / \mathrm{kg}$ natrium pentobarbital. The bulla was removed and opened and the ossicles were removed. Since a rabbit's malleus and incus are fused together, they had to be separated first. Afterwards, we performed the indentation tests on four positions as indicated in figure 7 . The measurements were perfomed within two hours post mortem. The results of these experiments are given in section 3.3. The study was performed according to the regulations of the local ethical committee. 


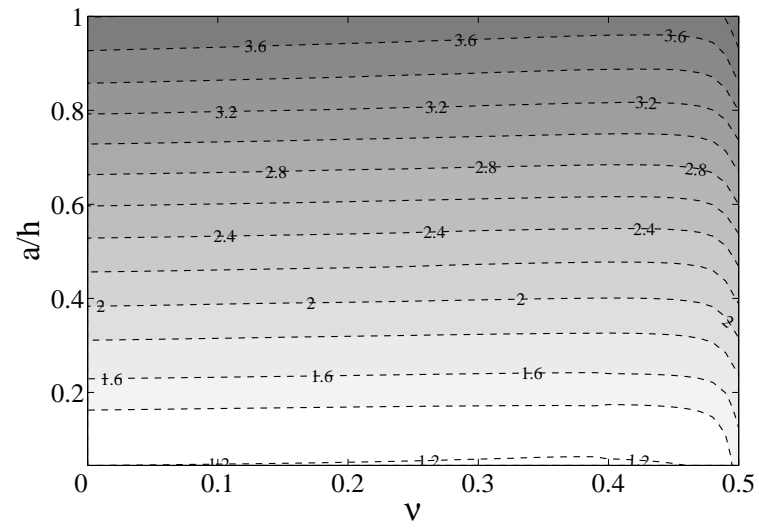

(a) frictionless model

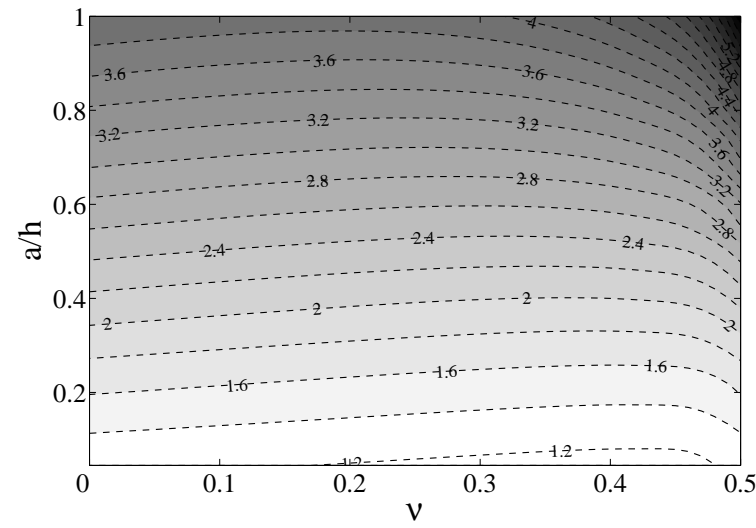

(b) adhesive interface condition

Fig. $5 \kappa$ calculated for different Poisson ratios $(\nu)$, and aspect ratio indenter radius and material thickness $(a / h)$. The contact zone between the indenter and the material surface is (a) a sliding contact, (b)fixed.

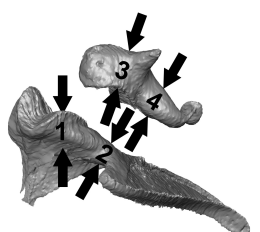

Fig. 7 Micro-CT reconstrunction of malleus and incudis with indentation positions indicated: 1.caput malleus, 2.collum malleus, 3.corpus incudis and 4.crus longum incudis

\section{Results}

\subsection{ASTM compression test}

In order to validate our new indentation setup, comparison needed to be made with standardised tests on large samples. Several experiments were performed for each material and the Young's modulus and its standard deviation were calculated. Since the results were very reproducible, only one example for each material is shown in figure 8. In order to have a clear graph, both stress and strain, which were negative in compression, are plotted as positive values. The compressive stiffness was calculated using the tangent line in the origin to the stress-strain curve.

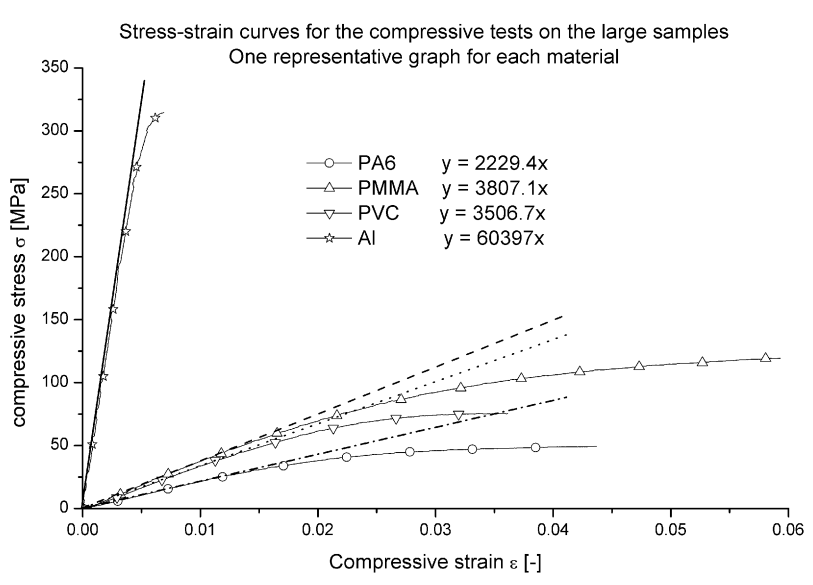

Fig. 8 Normal stress as function of the longitudinal strain for the four selected materials.

As can be seen in figure 8, the calculated Young's modulus varied between $2.2 \mathrm{GPa}$ and $60 \mathrm{GPa}$ for the four different materials, so the validation runs over a selected range of stiffness moduli. Table 1 gives an overview of all calculated Young's moduli and the standard deviation for all four materials.

Table 1 Elasticity modulus (in GPa, \pm stdev) obtained from ASTM D695-02a test.

\begin{tabular}{lcc}
\hline Material & $\mathrm{N}$ & $E_{A S T M}$ \\
\hline Al & 3 & $60.9 \pm 1.4$ \\
PVC & 5 & $3.526 \pm 0.061$ \\
PMMA & 5 & $3.799 \pm 0.039$ \\
PA6 & 5 & $2.260 \pm 0.0884$
\end{tabular}


Table 2 Elasticity moduli for four test-materials with different thickness, with $h$ the material thickness, $\nu$ the Poisson's ratio, $E_{A S T} M$ the elasticity modulus obtained with the standard compression test (table 1), $E_{S n e d d o n}$ the elasticity modulus obtained with equation $2, E_{\text {Indentation }}$ the corrected elasticity modulus \pm stdev (equation 4 ) and deviation $=\frac{E_{\text {indentation }}-E_{A S T M}}{E_{A S T M}}$.

\begin{tabular}{lcccccc}
\hline Material & $h(\mu \mathrm{m})$ & $\nu$ & $E_{\text {ASTM }}(\mathrm{GPa})$ & $E_{\text {Sneddon }}(\mathrm{GPa})$ & $E_{\text {indentation }}(\mathrm{GPa})$ & deviation \\
\hline Al & 975 & 0.33 & 60.9 & 76.9 & $64.6 \pm 5.5$ & $+6.1 \%$ \\
Al & 444 & 0.33 & 60.9 & 86.3 & $64.0 \pm 4.8$ & $+5.2 \%$ \\
Al & 200 & 0.33 & 60.9 & 117 & $66.9 \pm 6.7$ & $+9.9 \%$ \\
PVC & 988 & 0.35 & 3.526 & 3.87 & $3.27 \pm 0.14$ & $-7.2 \%$ \\
PVC & 440 & 0.35 & 3.526 & 4.32 & $3.21 \pm 0.17$ & $-8.9 \%$ \\
PVC & 300 & 0.35 & 3.526 & 4.77 & $3.21 \pm 0.26$ & $-9.0 \%$ \\
PMMA & 1010 & 0.40 & 3.799 & 4.97 & $4.23 \pm 0.22$ & $+11.4 \%$ \\
PMMA & 430 & 0.40 & 3.799 & 5.65 & $4.21 \pm 0.20$ & $+10.8 \%$ \\
PMMA & 275 & 0.40 & 3.799 & 6.50 & $4.27 \pm 0.22$ & $+12.6 \%$ \\
PA6 & 1030 & 0.39 & 2.260 & 2.70 & $2.30 \pm 0.14$ & $+1.8 \%$ \\
PA6 & 450 & 0.39 & 2.260 & 2.72 & $2.05 \pm 0.15$ & $-9.1 \%$
\end{tabular}

\subsection{Indentation test-material}

After performing the compression test, we used our new indentation method to determine the Young's modulus of the four test-materials. We examined specimens with different thicknesses ranging from $200 \mu \mathrm{m}$ to $1000 \mu \mathrm{m}$. For each test-case, three different samples were used and these were successively tested on three different indentation depths $(5 \mu \mathrm{m}, 7.5 \mu \mathrm{m}$ and $10 \mu \mathrm{m})$. These nine values enable us to calculate the average value and the standard deviation, which are shown in table 2 (in the column named $\left.E_{\text {indentation }}\right)$.

$E_{\text {Sneddon }}$, which is the Young's modulus calculated without the correctionfactor proposed in equation 4, is also given in table 2. Furthermore, the values of the sample thickness $h$, and the Poisson's ratio $\nu$ are also shown in this table. Finally, the deviation between these two techniques, defined as deviation $=\frac{E_{\text {indentation }}-E_{A S T M}}{E_{A S T M}}$, is also shown in table 2.

\subsection{Biological samples: middle ear ossicles}

After validating our technique on the test-materials, we used our new technique on biological samples with properties within the same range as the test-materials $(2 G P a<E<60 G P a$ and $200 \mu m<h<1000 \mu m)$.

After harvesting the malleus and the incus, four positions were carefully chosen (figure 7 : 1.caput malleus, 2.collum malleus, 3.corpus incudis and 4.crus longum incudis). On these precise locations the ossicles had reasonably parallel surfaces which meant they could be clamped between the two needles with a minimal nonflatness of the contact (figure 2). The entire process is followed with a light microscope.

After positioning the ossicles, the thickness, which was inevitably different for all locations, is measured using the indentation cones and the digital micrometer. The indentation measurements were done at depths between
$5 \mu m$ and $10 \mu m$ (in 3 or 4 steps) and on $N$ different specimens. The average and standard deviation for the Young's modulus found at different locations are presented in table 3 , together with the average thickness of the samples.

Table 3 The Young's moduli $( \pm s t d e v)$ for rabbit middle ear ossicles is obtained at different positions (caput malleus, collum malleus, corpus incudis and crus longum incudis) with different thickness (h). $\mathrm{N}$ is the number of different specimens.

\begin{tabular}{lccc}
\hline Position & $\mathrm{h}(\mu m)$ & Young's modulus $(\mathrm{GPa})$ & $\mathrm{N}$ \\
\hline caput malleus & 540 & $16.3 \pm 2.9$ & 5 \\
collum malleus & 480 & $15.6 \pm 1.8$ & 5 \\
corpus incudis & 770 & $16.8 \pm 3.1$ & 4 \\
crus longum incudis & 440 & $17.1 \pm 3.8$ & 3 \\
average & & $16.4 \pm 2.8$ & 17
\end{tabular}

\section{Discussion}

\subsection{FE corrected double indentation method}

In order to obtain the elasticity modulus for a material standard tensile tests are easy and fast to perform on large objects. Sample preparation and controlling the boundary conditions in such tests are difficult for smaller objects [3, 4]. Dedicated experiments to determine the modulus give very good results but are difficult to perform and are time consuming $[5,6,7]$.

Indentation tests, based on Sneddon's solution [8], do not need such complicated preparation and are easy to use on micro- and nanoscale. They are, however, designed for relatively thick materials which is an important drawback when applying the method to biomaterials: decreasing the needle radius to measure thinner objects can change the results drastically because the bulk properties are not measured anymore. This behaviour is even more important for biological materials which are often built up with cells and fibers [21]. Therefore 
we made two additions to Sneddon's solution.

First, we introduced a second needle to prevent surface deformations not caused by the indentation. By adding this second needle in such a way that a virtual mirror plane is introduced, the experimental setup becomes equivalent to a sample with half thickness which lays on a perfect sliding sample stage. Furthermore, the samples could be tested faster since they only had to be clamped between those two punches.

We should taken into account that we need to suppose that a perfect mirror plane develops in the specimen between the two indenters. This condition will only be met if the two indentation points are perfectly aligned. In order to acquire this condition, the loadcell is mounted on crossed translation tables with a translation precision of better than $1 \mu \mathrm{m}$. The indentation points are brought towards each other, and microscope observation from two perpendicular directions is used to align the indentation surfaces. The surfaces of the indentation points themselves are nearly perfectly perpendicular to the indentation direction. We achieved this by putting the cylindrical part of the points in a custom made holder which is placed perfectly perpendicular on a polishing disk, in order to polish the pointed end to a small plane which is perpendicular to the indentation axis. In addition the sample itself should be symmetrical on mechanical relevant places, which are the places with higher Von Mises stress in figure 4. Furthermore, an offset indentation is necessary to assure full contact between material and indenter surface. Otherwise, the stiffness will be underestimated, as seen in equation 3 . When the full contact requirements are not reached, in case of rough materials or inclined surfaces, sample preparation and polishing is needed. Observation with the light microscope allowed us to conclude that the bone samples which we used were smooth enough.

A second addition to Sneddon's solution was a new FE calculated correction factor $\kappa$ for those thin, freestanding materials. We take geometric nonlinearity, in contrast with the infinitesimal results of Hayes et al. [22] into account, but the finite deformation effect which causes stiffening during larger indentations was left out $[4,10]$. We obtained $\kappa$ values for a frictionless sliding contact and a fixed sliding contact, shown in figure 5 . The real values will be in between those two situations. In accordance with the conclusions by Zhang et al. [4], we could state that the value of $\kappa$ is fairly constant for Poisson's ratios between 0 and 0.4 , if the aspect ratio indenter radius and material thickness was smaller than 1 . The new $\kappa$-values are higher when compared with the values of Hayes et al. [22] and Zhang et al. [4], which were obtained for an elastic layer bounded to a rigid half space. The correction factor depends on the aspect ratio of the indenter radius and the material thickness $(a / h)$, and on the Poisson's ratio $(\nu)$. Considering a fixed or a sliding contact at the needle-surface zone gives approximately the same solution for $\kappa$ when the Poisson's ratio is smaller than 0.4 and aspect ratio is smaller than 1, which corresponds well with the conclusions by Zhang et al. [4].

By using a more sensitive loadcell, our technique can possibly also be extended towards much softer materials such as biological tissue. Because the indentation surfaces are very small, there will be little effect from local bending on the indentation surface itself. A very soft material should be supported in a holder to prevent the material from bending around the needle. In addition, an active vibration isolation will be necessary when smaller forces need to be measured. However, from our calibration measurements we did see that no significant noise was picked up due to acceleration effects caused by external vibrations. Therefore a regular rigid table was sufficient for our setup. Providing such technical problems can be solved we think our method can have promising applications in soft tissue testing as well, but at the moment we are not equipped to demonstrate this.

Thinner objects could be measured by using a different indenter radius, but this will also change the physical contact-properties as remarked earlier. In that case $\kappa$ should be tested for thinner materials. The bulk properties of thicker materials can also be determined with this method. Furthermore, larger indentation punches are produced more easily. When the aspect ratio of the indenter radius and the material thickness $(a / h)$ is smaller than 0.025 , the correction factor $\kappa$ will be lower than 1.1. The double indentation will still be necessary to ensure no unwanted surface deformations. When the sample dimensions are larger than a few millimeters, tensile tests can easily be applied to determine material bulk properties. The materials which we examined with this indentation test, were considered to be homogenous, isotropic and linear (assumption in Sneddon's solution and in the FE calculations), just like in the ASTM test.

\subsection{Loading protocol}

Visco-elastic materials showed creep and relaxation and there were some plastic deformations and phase transformations due to local high stresses [12, 17]. Also, hysteresis caused the load and unload curves to be different. In order to minimize these effects, pre-loadings were used and the indentation-force curve was measured during unloading [20]. The force-indentation curves 
for most materials showed increasing stiffness for increasing indentation depth. This effect could be attributed to nonlinear phenomena, but it is also the result from geometric nonlinearity $[4,10]$. The indentation depths should not be too deep to minimize this effect. Therefore, several offset indentations had to be tested empirically and the loading protocol as presented in figure 3 was introduced.

\subsection{Validation}

In order to test the proposed method, four test-materials with different material-properties (Al, PVC, PMMA and PA6) were selected and tested according to the ASTM D695-02a standard. The standard deviation calculated from different samples was between 1 and $4 \%$. A second part of the four test-materials were prepared with different thicknesses between $200 \mu \mathrm{m}$ and $1000 \mu \mathrm{m}$ and were used for the new double indentation method. As such, the FE calculated correction factor $\kappa$ and the thickness independency of the measurement could be tested. A Young's modulus $E_{\text {indentation }}$ and a standard deviation, which ranged between 5 and $10 \%$, is obtained and presented in table 2. The Poisson's ratio, used in the calculations, were also presented. It should be possible to measure the Poisson's ratio by using two different sizes of indentation punches [28].

When comparing the results from the standard compression method $\left(E_{A S T M}\right)$ and from our double-needle indentation method ( $\left.E_{\text {indentation }}\right)$, the difference between the results obtained from the two techniques was always smaller than $13 \%$ (table 2). We also demonstrated the importance of the correction factor $\kappa$ for thin materials by calculating $E_{S n e d d o n}$. The correction factor goes up to 1.75 for thin materials and the difference between the results of our method and Sneddon's solution goes up to $92 \%$. From the validation measurements we learn that our method allows to determine Young's moduli to an accuracy of better than $13 \%$ on small specimens in a large range of Young's moduli and material thickness $(2 G P a<E<60 G P a$ and $200 \mu m<h<1000 \mu m)$.

\subsection{Biological materials}

In current mechanobiological research linear FE models are often used. Such models need information about the geometry, the boundary conditions and the material properties of all parts. The most important parameter for linear elastic models is the Young's modulus. Often general parameters are used: for instance for bone, the values of Evans [2] which were measured on the unembalmed wet cortical bone of the human femur are used even in papers dealing with other species or other types of bone[23]. In our method we only measure the bulk elasticity parameter of the material. Biological materials often consist of fine structures, which we do not take into account. However, the results from our method are intended to be used in FE modelling of biomechanical structures, where materials are often approximated as being homogeneous. Nevertheless, great care should always be taken, as in some cases the outer surface of a biological object can have significantly different properties from its inner structure. With our method one can harvest thin samples out of such a structure, and thus obtain more detailed information on the distribution of the elasticity parameters, this is not possible in a classical compression test. It remains impossible to take into account inhomogenities within the thin sample itself.

Rabbit middle ear ossicle bone is a good example of the usefulness of our method in the mechanobiology, since we know that the Young's modulus of bone should be in the range of the test-materials [2] and since middle ear ossicle bone is too small for standard test methods [3]. In hearing science [23, 24, 29, 30], proper models with correct material-parameters are important to investigate the functioning of normal and pathologic ears $[31,32]$ by describing the correct ossicle bending and so their results for the middle ear transfer function $[25,26]$. On incus and malleus, 4 locations are found with approximately parallel surfaces so the indentation test could be performed easily. The thickness cannot be chosen freely, but was measured and it was in the same range as the thickness of the samples used in the validation experiments. Young's moduli for all bones were found between 11 and $22 \mathrm{GPa}$, which is in the range of the test-materials. Standard deviations, which are obtained from indentation on different depths (between 5 and $10 \mu \mathrm{m}$ ) and on different specimens, ranged from 10 to $22 \%$. When comparing the elasticity modulus between different locations no significant difference was found and the Young's modulus from rabbit middle ear ossicle bone could be determined as $16 \pm 3 \mathrm{GPa}$ which is higher than the value given by Evans [2] (12 \pm 3 $\mathrm{GPa}$. When we compared this result to the nanoindentation results obtained by Rho et al. [33], we found that our value was between the highest and lowest Young's modulus of the microstructural components of bone (13.4GPa for trabecular and 25.8GPa for cortical bone). These microstructural properties are very useful for a better understanding of the building up and functional behaviour of bone, but for modelling of complex biomechanical systems, such as the middle ear, a general bulk parameter will mostly suffice. 


\section{Conclusion}

We have developed a portable double indentation device which allows to measure the Young's modulus of thin samples. The material is clamped between two needles and symmetrically indented. As such, the problem of creating a perfect smooth contact zone between sample and sample stage is avoided, as a virtual plane is created due to symmetry. A correction factor $\kappa$, which compensates for the small thickness, is calculated with FE modelling and added to Sneddon's solution. As such, the Young's modulus of the validation materials could easily be measured, independent from thickness, with an accuracy better than $13 \%$. As a demonstration, we applied our technique on the small ME ossicle bone of rabbits and found a Young's modulus of $16 \pm 3 \mathrm{GPa}$.

Acknowledgements Financial support to this project is given by the Research Foundation - Flanders (FWO). We thank F. Wiese, J. Van Daele and W. Deblauwe for their technical assistance, V. Vandervelden and B. Soons for proofreading the english writing and J. Buytaert for his 3D model of the middle ear ossicles (www.ua.ac.be/bimef).

\section{References}

1. Strait DS, Wang Q, Dechow PC, Ross CF, Richmond BG, Spencer MA, Patel BA. Modeling elastic properties in finite-element analysis: how much precision is needed to produce an accurate model? Anat Rec A Discov Mol Cell Evol Biol, 283(2):275287, Apr 2005.

2. Evans FG. Mechanical properties of bone. Thomas Springfield, IL, 1973.

3. Speirs AD, Hotz MA, Oxland TR, Husler R, Nolte LP. Biomechanical properties of sterilized human auditory ossicles. J biomech, 32:485-491, 1999.

4. Zhang M, Zheng YP, Mak AFT. Estimating the effective youngs modulus of soft tissues from indentation tests nonlinear finite element analysis of effects of friction and large deformation. Med Eng Phys, 19:512-517, 1997.

5. Kvistedal YA, Nielsen PMF. Estimating material parameters of human skin in vivo. Biomechan Model Mechanobiol, 8:1-8, 2009.

6. Meunier L, Chagnon G, Favier D, Orgas L, Vacher P. Mechanical experimental characterisation and numerical modelling of an unfilled silicone rubber. polym test, 27:765-777, 2008.

7. Samani A, Plewers D. A method to measure the gyperelastic parameters of ex vivo breast tissue samples. Phys med biol, 49:4395-4405, 2004.

8. Sneddon IN. The relaxation between load and penetration in the axisymmetric boussinesq problem for a punch of arbitrary profile. Int $j$ Eng Sci, 3: 47-57, 1965.

9. Bolduc JE, Lewis LJ, Aubin CE, Geitmann A. Finite-element analysis of geometrical factors in micro-indentation of pollen tubes. Biomech Model Mechanobiol, 5(4):227-236, Nov 2006.

10. Choi APC, Zheng YP. Estimation of youngs modulus and poissons ratio of soft tissue from indentation using two different sized indentors: finite element analysis of the finite deformation effect. Med Biol Eng Comput, 43:258-264, 2005.

11. Feng C, Tannenbaum JM, Kang BS, Alvin MA. A load-based multiple-partial unloading microindentation technique for mechanical property evaluation. exp mech, 2009. doi: 10.1007/s11340-0099271-4.

12. Galanov BA, Domnich V, Gogotsi Y. Elasticplastic contact mechanics of indentations accounting for phase transformations. exp mech, 43:303308, 2002.

13. Ju BF, Ju Y. Video enhanced depth-sensing indentation technique for charaterizing mechanical behaviour of biomaterials,. Meas sci technol, 17:17761784, 2006.

14. Lin D, Shreiber D, Dimitriadis E, Horkay F. Spherical indentation of soft matter beyond the hertzian regime: numerical and experimental validation of hyperelastic models. Biomech Model Mechanobiol, Nov 2008.

15. Riccardi B, Montanari R. Indentation of metals by a flat-ended cylindrical punch. Mater Sci Eng A, 381:281-291, 2004.

16. Gong J, Miao H, Peng Z. Simple method for determining the initial unloading slope for ceramics nanoindentation tests. J mater sci lett, 22:267-267, 2003.

17. Huang G, Lu H. Measurements of two independent viscoelastic functions by nanoindentation. exp mech, 47:87-98, 2006.

18. Scholz I, Baumgartner W, Federle W. Micromechanics of smooth adhesive organs in stick insects: pads are mechanically anisotropic and softer towards the adhesive surface. J Comp physiol A, 194: 373-384, 2008.

19. Shuman DJ, Costa ALM, Andrade MS. Calculating the elastic modulus from nanoindentation and microindentation reload curves. Mater charact, 58: 380-389, 2007.

20. Oliver WC, Pharr GM. An improved technique for determining hardness and elastic modulus using load and displacement sensing indentaion experiments. mater res, 7:1564-1583, 1992. 
21. Choi K, Kuhn JL, Ciarelli MJ, Goldstein SA. The elastic moduli of human subchondral, trabecular, and cortical bone tissue and the size-dependency of cortical bone modulus. J Biomech, 23(11):11031113, 1990.

22. Hayes WC, Keer LM, Herrmann G, Mockros LF. A mathematical analysis for indentaion tests of articular cartilage. J. Biomec, 5:541-551, 1972.

23. Koike T, Kobayashi T, Wada H. Modeling of the human middle ear using finite-element method. $J$ Acoust Soc Am, 111 (3):1306-1317, 2002.

24. Sun Q, Gan RZ, Chang KH, Dormer KJ. Computer-integrated finite element modeling of human middle ear. Biomechan Model Mechanobiol, 1: 109-122, 2002.

25. Decraemer WF, Shyman MK, Funnell WRJ. Malleus vibration mode changes with frequency. hear res, 54:305-318, 1991.

26. Dirckx JJJ, Buytaert JAN, Decraemer WF. Quasistatic transfer function of the rabbit middle ear, measured with a heterodyne interferomenter with high resolution position decoder. JARO, 7 (4):339351, 2006.

27. Maas S, Weiss JA. Febio: Finite elements for biomechanics. user's manual, version 1.0. Online publication, 2008.

28. Jin H, Lewis JL. determination of poissons ratio of articular cartilage in indentation test using different sized indenters. In Summer bioengineering conference, Florida, 2003.

29. Decraemer WF, Dirckx JJJ, Funnell WRJ. Threedimensional modelling of the middle-ear ossicular chain using a commercial high-resolution x-ray ct scanner. J Assoc Res Otolaryngol, 4(2):250-263, Jun 2003.

30. Dirckx JJJ, Decraemer WF, von Unge M, Larsson C. Measurement and modeling of boundary shape and surface deformation of the mongolian gerbil pars flaccida. Hear Res, 111(1-2):153-164, Sep 1997.

31. Dirckx JJJ, Decraemer WF. Effect of middle ear components on eardrum quasi-static deformation. Hear Res, 157(1-2):124-137, Jul 2001.

32. von Unge M, Decraemer WF, Dirckx JJJ, BaggerSjbck D. Tympanic membrane displacement patterns in experimental cholesteatoma. Hear Res, 128 (1-2):1-15, Feb 1999.

33. Rho JY, Tsui TY, Pharr GM. Elastic properties of human cortical and trabecular lamellar bone measured by nanoindentation. Biomaterials, 18(20): 1325-1330, Oct 1997. 